\title{
Alle reden über Blockchain
}
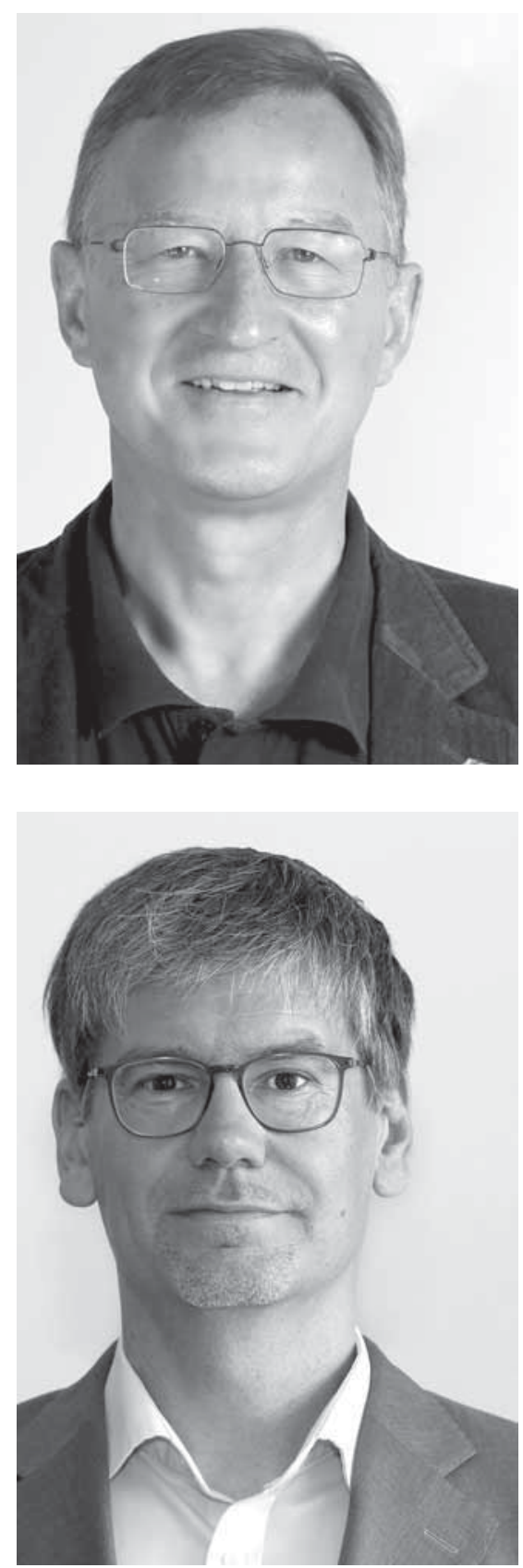

Typisch ist das kürzlich im ICE unfreiwillig mitgehörte Gespräch eines mobil managenden Mitreisenden, der in sein Handy rief:, „... Blockchain, ja ich rufe wegen Blockchain an, das ist doch die Technologie, die gerade sämtliche Branchen umkrempelt." Tatsächlich gibt es nicht wenige Experten, die der Blockchain-Technologie einen Innovationsschub zutrauen wie seinerzeit bei der Einführung des Internet. Viele warnen davor, etwas Entscheidendes zu verpassen, wenn man nicht rechtzeitig dabei ist.

Diesem Hype gehen wir mit diesem Schwerpunktheft auf den Grund. Dabei registrieren wir die drei grundlegenden Eigenschaften, die die Blockchain-Technik seit dem 2009 eingeführten Bitcoin-Verfahren zum pseudonymen Bezahlen auszeichnen: Pseudonymität, dezentrale und transparente Vertrauensverteilung zwischen den Teilnehmern und die Sicherheit der Transaktionsdaten. Ob diese Eigenschaften ausreichend sind, den Datenschutz zu revolutionieren und gleichzeitig neue Anwendungen kostengünstig zu befördern, untersuchen wir hier anhand eines analytischen Blicks auf die Prinzipien des Datenschutzes und auf bestehende Anwendungen bzw. Anwendungsvorstellungen.

Rainer Böhme von der Universität Innsbruck und Paulina Pesch vom Karlsruher Institut für Technologie (KIT) legen die Basis unserer Betrachtungen mit ihrem Beitrag über die technischen Grundlagen und die datenschutzrechtlichen Fragen der Blockchain-Technologie. Stephan Wiefling, Frederik Sandbrink und Luigi Lo lacono von der Technischen Hochschule Köln stellen auf dieser Grundlage einige Anwendungen der Blockchain außerhalb von Geldwährung vor, indem sie die zugehörige Literatur auswerten. Das Anwendungsfeld der sogenannten Smart Contracts untersuchen Christian Sillaber (Universität Innsbruck) und Bernhard Waltl (Technische Universität München). Der Beitrag von Martin Grottenthaler und Jürgen Fuß von der Fachhochschule Hagenberg in Oberösterreich beschreibt die Anwendung der Blockchain-Technologie auf die Absicherung von E-Mails. Nadine Rückeshäuser, Christian Brenig und Günter Müller von der Universität Freiburg untersuchen schließlich, welche digitalen Geschäftsmodelle in welcher Weise für Blockchains überhaupt taugen.

Diesem Schwerpunktheft liegen die Beiträge eines Arbeitsworkshops zugrunde, den die GI-Fachgruppe E-Commerce und E-Government (ECOM) des Fachbereichs Sicherheit zusammen mit dem Fraunhofer-Institut SIT und der Zeitschrift Datenschutz und Datensicherheit (DuD) am 20. April 2017 in Darmstadt veranstaltet hatte (https:// www.sit.fraunhofer.de/de/news/landingpages/gi-ecom-workshop/). Die Erkenntnisse aus diesem Heft werden darüber hinaus auf einem Workshop des CAST e.V. am 26. Oktober 2017 (https://www.cast-forum.de/) vorgestellt.

\section{Rüdiger Grimm und Andreas Heinemann}

